\title{
Aneurysm and Artery Dissection Following the use of Vascular Endothelial Growth Factor Inhibitor: A Real-World Analysis Using a Spontaneous Reporting System
}

Jian Gong ( $\nabla$ gongjian_1979@163.com )

Shenyang Pharmaceutical University

WANG Shuyue

Shenyang Pharmaceutical University

CHEN Mingzhu

Shenyang Pharmaceutical University

ZHANG Xinghui

Shenyang Pharmaceutical University

ZHANG Lingjian

Shenyang Pharmaceutical University

JIA Min

Shenyang Pharmaceutical University

\section{SHEN Zhiwen}

Shenyang Pharmaceutical University

WANG Junyan

Shenyang Pharmaceutical University

$X U$ Jing

Shenyang Pharmaceutical University

\section{ZHENG Zheng}

Shenyang Pharmaceutical University

WANG Mengmeng

Shenyang Pharmaceutical University

LV Xuanrui

Shenyang Pharmaceutical University

\section{ZONG Xiaoyu}

Shenyang Pharmaceutical University

LI Hui

Shenyang Pharmaceutical University

ZHOU Jin

Shenyang Pharmaceutical University 


\section{MENG Tong}

Shenyang Pharmaceutical University

\section{Bin Zhao}

Peking Union Medical College Hospital

\section{GONG Jian}

Shenyang Pharmaceutical University

\section{Research article}

Keywords: VPI, disproportionality analysis, pharmacovigilance, cancer

Posted Date: December 2nd, 2020

DOl: https://doi.org/10.21203/rs.3.rs-115864/v1

License: (c) (i) This work is licensed under a Creative Commons Attribution 4.0 International License.

Read Full License 


\section{Abstract}

Background: There are few real-world studies comparing the incidence, clinical features, and prognosis of aneurysm and artery dissection related to various vascular endothelial growth factor inhibitor (VPI) regimens. This study evaluated the recent evidence of aneurysm and artery dissection after VPI therapy. The purpose was to evaluate and compare the links between different VPIs and aneurysm and artery dissection by investigating the Food and Drug Administration Adverse Event Reporting System (FAERS).

Methods: Using FAERS data from January 2004 to March 2020, disproportionality analysis was used for data mining to screen suspected aneurysm and artery dissection cases after different VPI treatments. The times to onset and fatality rates of suspected drugs were also investigated to assess prognoses.

Results: In total, there were 634 reports of aneurysm and artery dissection events after VPI. Ramucirumab appeared to have a stronger association than other VPIs, based on the reporting odds ratio $(\mathrm{ROR}=3.68$, $95 \%[2.18,6.23])$. The results showed a significant difference in onset time $(P<0.001)$, and a quick onset occurred in $60.13 \%$ of cases. The median time to aneurysm and artery dissection was 79.5 (IQR: 19273.5) days after VPI administration. The results also showed that men were more affected than women $(59.68 \%$ vs. $40.32 \%)$, and patients older than 45 years were more affected than younger patients $(68.12 \%$ vs. $3.93 \%$ ). Finally, the suspected drugs generally led to a $19.98 \%$ fatality rate and a $29.81 \%$ hospitalization rate. The highest death rate was associated with Aflibercept (25.49\%).

Conclusions: We identified signals for aneurysm and artery dissection following various VPIs in real-world practice using the FAERS, which represents the first step for continued pharmacovigilance investigation.

\section{Introduction}

Vascular endothelial growth factor (VEGF) plays an important role in physiological and pathological angiogenesis. Interactions between VEGF and VEGF receptors expressed on endothelial cells lead to increased normal blood vessel proliferation, migration, degeneration, and permeability. Pharmacological inhibition of angiogenesis via the VEGF pathway is an important therapeutic target that prevents tumor growth and the formation of metastases [1,2]. Anti-VEGF therapies currently approved for use in various types of cancer include small molecule tyrosine kinase inhibitors that target multiple molecular pathways, including monoclonal antibodies, fusion proteins, and VEGF itself. Until recently, there were 24 commercially available vascular endothelial growth factor pathway inhibitors (VPIs) (supplementary table 1). The most common side effects of drugs that target the VEGF pathway are hypertension and proteinuria [3]. However, the use of anti-VEGF agents has been reported to have more serious side effects such as aneurysms and aortic dysfunction [4-7]. Reports of aneurysms and dissections associated with VPI treatments have been reported from 2008 onwards [8]. However, most evidence comes from the Pharmaceutical and Medical Devices Agency rather than clinical cohorts or case-control studies [8-10], which is insufficient to understand relatively rare adverse events. There has been no pharmacovigilance study until recently explaining VPI-mediated aneurysms and arterial dissections, and knowledge of the 
vascular safety profile following various VPIs in actual clinical practice is poor. Therefore, the purpose of this study was to evaluate and compare the associations between various VPIs and aneurysms and dissections by investigating the Food and Drug Administration's (FDA) Adverse Event Reporting System (FAERS). In this study, we also investigated mortality rates and the time to onset of aneurysms and arterial dissections for various VPI regimens.

\section{Methods}

Data source

This retrospective pharmacovigilance study was conducted using data obtained from the FAERS database from January 2004 to March 2020. The FAERS database is a public and voluntary reporting system (SRS) that contains information on adverse drug events and medication error reports submitted by health experts, patients, and manufacturers in the United States and other regions. FAERS data include eight datasets that cover everything needed for pharmacovigilance research $[11,12]$. According to FDA recommendations, we reduced the number of reports to 634 by choosing the latest FDA_DT if the CASEIDs were the same and the higher PRIMARYID if the CASEIDs and FDA_DTs were the same.

Adverse events and drug identification

We used MedDRA (Version 23.0) Preferred Term "aneurysms and artery dissections" (code: 10002363) to investigate adverse events in the REAC files (See Supplementary Table 2 for the complete search strategy of PTs). In the data mining process, IBM Micromedex (IBM Corp., Armonk, NY, USA) was used as a dictionary to select the generic and brand names of VPIs.

Data mining

The data mining algorithm used for this analysis was the report odds ratio (ROR) and corresponding 95\% confidence intervals (Cls) for the association between the use of VPIs and aneurysms and artery dissection. A value of ROR-1.96SE $>1, N>2$ was considered as positive signal strength [13-15]. We evaluated the time to onset of aneurysms and artery dissections by defining the interval between the onset date of adverse events and the start date of VPI therapy. We also analyzed reports of fatal events due to side effects and calculated mortality as the total number of VPI-induced aneurysm or dissection events.

\section{Statistical analysis}

Descriptive analysis was used to summarize the clinical features of patients with aneurysm and artery dissection. The onset time of VPI-related aneurysms and arterial dissections between different VPIs was compared using the Kruskal-Wallis test and Dan's multiple comparison test. Mortality rates were compared between different VPIs using Pearson's Chi-square test or Fisher's exact test. Statistical 
significance was set to $\mathrm{P}<0.05$ with a $95 \%$ confidence interval. All statistical analyses were performed using GraphPad Prism 8 (GraphPad Software Inc., San Diego, CA, USA).

\section{Results}

General characteristics

In total, there were 634 total reports of VPI-related aneurysm and artery dissection, among which, 497 (78.39\%) were submitted by healthcare professionals, 115 (18.14\%) were submitted by consumers, leaving $22(3.47 \%)$ cases with unspecified reporters.

The clinical features of these patients are shown in Table 1 and Supplementary Table 3. The number of reported cases of VPI-related aneurysm and artery dissection showed a gradually increasing trend from 2004 to 2020, peaking at 92 cases (14.51\%) in 2018. The data were collected from six regions and 43 countries. There were $225(35.50 \%)$ cases from Asia, followed by $223(35.17 \%)$ and $146(23.04 \%)$ from North America and Europe, respectively. By country, 203 (32.02\%) cases were reported from Japan (Asia), followed by 201 (31.7\%) from USA (North America) and 28 (4.42\%) from France (Europe). By type of VPI, the highest number of aneurysm and artery dissection reports were from bevacizumab 223 (35.57\%), followed by ranibizumab 104 (16.59\%) and sunitinib 96 (15.31\%). VPIs are suitable for various tumor types, and the most common cases in this study were renal cancer patients $(n=115,18.86 \%)$. Excluding cases of unspecified age, the mean age of patients was 67.43 years. Patients aged $\geq 45$ years were more affected than younger patients ( $n=312,68.12 \%$ vs. $n=18,3.93 \%$ ); patients aged $\geq 85$ years accounted for $5.46 \%(n=25)$ of reported cases. Excluding the unspecified data, men were reported more than women $(n=336,59.68 \%$ vs. $n=227,40.32 \%)$. 
Table 1

Characteristics of cases with VPIassociated aneurysm and artery dissection

\begin{tabular}{|c|c|}
\hline Characteristics & Reports, no. (\%) \\
\hline \multicolumn{2}{|l|}{ Reporter } \\
\hline consumer & $115(18.14)$ \\
\hline health-professional & 497 (78.39) \\
\hline unspecified & $22(3.47)$ \\
\hline \multicolumn{2}{|c|}{ VEGFI as suspected drugs } \\
\hline Sorafenib & $38(6.06)$ \\
\hline Ponatinib & $8(1.28)$ \\
\hline aflibercept & $33(5.26)$ \\
\hline pegaptanib & $1(0.16)$ \\
\hline Nintedanib & $23(3.67)$ \\
\hline axitinib & $13(2.07)$ \\
\hline bevacizumab & $223(35.57)$ \\
\hline ramucirumab & $14(2.23)$ \\
\hline ranibizumab & $104(16.59)$ \\
\hline brolucizumab & $2(0.32)$ \\
\hline Sunitinib & $96(15.31)$ \\
\hline regorafenib & $8(1.28)$ \\
\hline vandetanib & $2(0.32)$ \\
\hline pazopanib & $20(3.19)$ \\
\hline lenvatinib & $31(4.94)$ \\
\hline cabozantinib & $11(1.75)$ \\
\hline \multicolumn{2}{|l|}{ Age groups (years) } \\
\hline$<18$ & $1(0.22)$ \\
\hline $18-44$ & $17(3.71)$ \\
\hline $45-64$ & $145(31.66)$ \\
\hline $65-74$ & 167 (36.46) \\
\hline
\end{tabular}




\begin{tabular}{|ll|}
\hline Characteristics & Reports, no. (\%) \\
\hline $75-84$ & $103(22.49)$ \\
$>85$ & $25(5.46)$ \\
\hline Gender & \\
\hline Female & $227(40.32)$ \\
Male & $336(59.68)$ \\
\hline
\end{tabular}

Disproportionality analysis and Bayesian analysis

The signal intensity of $16 \mathrm{VPI}$ drugs with aneurysm and artery dissection was calculated by the RORs algorithm (Table 2). Only Sorafenib, Sunitinib, Lenvatinib, Ponatinib, Nintedanib, Bevacizumab, Ramucirumab, and Ranibizumab showed positive signal strength, and the signals showed that the correlation intensity between adverse reactions and the reported drugs were generally low. 
Table 2

Aneurysm and artery dissection signals based on the four algorithms

\begin{tabular}{|lll|}
\hline & N & ROR \\
\hline sorafenib & 38 & $(95 \%$ two-sided CI) \\
\hline axitinib & 14 & $1.25(1.02,1.93)^{*}$ \\
\hline apatinib & 0 & - \\
\hline sunitinib & 99 & $1.93(1.59,2.36)^{*}$ \\
\hline regorafenib & 8 & $0.79(0.39,1.57)$ \\
\hline vandetanib & 2 & $1.57(0.39,6.29)$ \\
\hline pazopanib & 20 & $0.61(0.39,0.95)$ \\
\hline lenvatinib & 31 & $3(2.11,4.28){ }^{*}$ \\
\hline cabozantinib & 12 & $0.61(0.34,1.07)$ \\
\hline Ponatinib & 8 & $2.03(1.01,4.06)^{*}$ \\
\hline aflibercept & 33 & $1.21(0.86,1.70)$ \\
\hline fruquintinib & 0 & - \\
\hline pegaptanib & 1 & $1.49(0.21,10.64)$ \\
\hline tivozanib & 0 & - \\
\hline brivanib & 0 & - \\
\hline conbercept & 0 & - \\
\hline linifanib & 0 & - \\
\hline nintedanib & 25 & $2.12(1.43,3.14)^{*}$ \\
\hline motesanib & 0 & - \\
\hline cediranib & 0 & - \\
\hline bevacizumab & 223 & $3.05(2.67,3.48){ }^{*}$ \\
\hline ramucirumab & 14 & $3.68(2.18,6.23)^{*}$ \\
\hline ranibizumab & 104 & $3.39(2.80,4.11)^{*}$ \\
\hline & & \\
\hline * & & \\
\hline
\end{tabular}

*The results were considered positive signal strength. 


\begin{tabular}{|lcc|}
\hline & N & ROR \\
\hline brolucizumab & 2 & $2.48(0.62,9.94)$ \\
\hline *The results were considered positive signal strength. \\
\hline
\end{tabular}

Time to onset of VPI-associated aneurysm and artery dissection

The median time to onset of VPI-related aneurysm and artery dissection was 79.5 days (IQR: 19-273.5). The time to onset of aneurysm and artery dissection for each VPI are described in Fig. 1. It was noteworthy that after the first dose of any VPI regimen, aneurysm and artery dissection could potentially occur. Rapid onset of aneurysm and artery dissection took place in $60.13 \%(n=184)$ of all VPI-associated aneurysm and artery dissection events. There was a significant difference between the various VPI treatments (Kruskal-Wallis test, $P<0.001$ ), with a minimum median time of 13.5 days (IQR: $3-59)$ for Regorafenib and a maximum of 494.5 days (IQR: 60.25-1000) for Ponatinib.

Fatalities due to VPI-associated aneurysm and artery dissection

The prognoses of VPI-related aneurysm and artery dissection cases were evaluated by mortality from adverse vascular events after various VPI treatments (Fig. 2). VPI-related aneurysm and artery dissection generally had a mortality rate of $19.98 \%(n=185)$. Among all VPIs, Ponatinib vs. Aflibercept and Aflibercept vs. Regorafenib had a significant difference in mortality (Pearson's chi-squared test for overall comparison, $P<0.05)$. The results also showed that there were zero deaths in patients who were administered Ponatinib, Pegaptanib, Regorafenib, and Vandetanib.

\section{Discussion}

To the best of our knowledge, this study represents the first and largest collection of links, timing, and prognosis for aneurysm and artery dissection after using various VPIs. Moreover, these data are from real-world practice based on the FAERS. Only $50 \%$ of drugs that showed VPI-related aneurysm and artery dissection had harmful positive signals. The highest signal of aneurysm and artery dissection reports was for Ramucirumab (ROR: 3.68, 95\%Cl: 2.18-6.23), followed by Ranibizumab (ROR: 3.39, 95\%Cl: 2.84.11) and Bevacizumab (ROR: 3.05, 95\%Cl: 2.67-3.48); Bevacizumab was the first VPI approved by the FDA in 2004, but four years later (2008) Aragon-Ching et al. considered it to be a drug potentially related to aortic dissection.[8]. Until now, there have been reports of aneurysms and arterial dissections caused by use of VPIs, but these have primarily been case reports [5, 6, 8-10, 16-22]. Due to limited sample size, relatively low incidence, and high confounding factors, there is still not enough power to draw clear conclusions about drug safety. Besides, it is also a challenge to evaluate and characterize it through persuasive randomized controlled trials (RCT).

Based on the FAERS system, reports of VPI-related aneurysm and artery dissection events are increasing annually. Among the statistical results, $18.14 \%$ of the reports were provided by consumers. This 
phenomenon indicated that VPI-associated aneurysm and artery dissection are being gradually recognized. Our results also indicated that VPI-associated aneurysm and artery dissection more commonly affected middle-aged and elderly patients and more men than women. Although there have been reports that VPI therapy can cause severe vascular damage, its exact role in the initiation of aneurysms and arterial dissections remains unclear [6].

In this pharmacovigilance study, not all VPIs were associated with aneurysm and artery dissection, but Ramucirumab had the strongest association among all other VPIs. In contrast, Bevacizumab showed a relatively weak association; however, cases of Bevacizumab-induced aneurysm and artery dissection have received attention in clinical practice $[5,7,8,10,23]$. Of course, this does not rule out the confounding effects of Bevacizumab on hypertension. Regrettably, clinical studies still lack a head-tohead comparison of the effects on the vasculature between different VPIs. Another major finding is that the median time to onset of vascular effects was 79.5 days (IQR: 19-273.5) between VPI regimens, with approximately $60.13 \%(n=184)$ of patients rapidly experiencing aneurysm and artery dissection after the first use. Therefore, once VPI is initiated, monitoring of vascular function is required, at least in sensitive patients. Diversity in the mean onset time between VPI regimens suggests that individualized monitoring strategies can be performed after VPI administration. In particular, observing vascular function immediately after applying Regorafenib and regularly assessing the need for long-term VPI use to avoid possible harm will be critical modifications.

Mortality and hospitalization rates were investigated to further compare the severity of aneurysm and artery dissection associated with various VPIs. The results showed that aneurysm and artery dissection generally led to a $29.81 \%(n=276)$ hospitalization rate and a $19.98 \%(n=185)$ mortality rate. The deaths associated with Regorafenib-induced aneurysm and artery dissection remained near zero, but the hospitalization rate for the same set was ranked highest at $53.33 \%(n=8)$. Indeed, the number of Regorafenib representatives was not as strong as a number of other VPIs in this study. The hospitalization rate for Ranibizumab, which had a clear adverse signal according to pharmacovigilance, was $17.69 \%(n=23)$ and the mortality rate was $25.38 \%(n=33)$. These data may indicate that users of Ranibizumab required more intensive care after the onset of the aneurysm and artery dissection. These findings can be applied in the clinic to determine the best VPI treatment option, taking into account the various VPI trends for patient age, gender, vascular function, and aneurysm to identify patients at highrisk of aneurysms and arterial dissections.

Although there are advantages of real-world research and data mining techniques in this study, it must be acknowledged that certain analyses of drug side effect signals are not feasible based on SRS. Therefore, this study had certain limitations. First, there are limitations to using the FAERS database. These data can be voluntarily reported by consumers, which could cause reporting bias and noise. Also, data on possible confounding factors were not systematically collected, including patient background information, potential conditions, concomitant drugs, and time to treatment. These are especially important for patients who are susceptible due to aberrant blood vessels from VPI treatment. Second, Vascular function declines with age. Based on the published medical records, VPI-associated aneurysm and artery 
dissection were most commonly accompanied by vascular diseases such as hypertension. The effects of these confounding exposures can disguise their contribution to VPI vascular toxicity. Third, there were a number of missing or unknown data in our collection, and it would be premature to draw a firm conclusion given this limitation. Although FAERS has some inherited limitations, it revealed important aspects of VPI-related aneurysm and artery dissection, providing clues for a more elaborate design research.

\section{Conclusions}

In this study, we identified factors associated with aneurysm and artery dissection following treatment with various VPIs in actual practice based on the FAERS database. One finding from this study was that not all VPIs are associated with aneurysm and artery dissection. Based on the ROR algorithm, only Sorafenib, Sunitinib, Lenvatinib, Ponatinib, Nintedanib, Bevacizumab, Ramucirumab had a stronger association between aneurysms and dissections. There was also a significant difference in the time to onset of the aneurysm and artery dissection after different VPIs, which should be immediately noted after the first dose of the VPI regimen. Finally, we found that older men were generally more sensitive to VPIrelated aneurysm and artery dissection. Our findings are the first step in an ongoing pharmacovigilance study that will encourage further research to test the hypotheses generated in this study.

\section{Abbreviations}

VPI(s)

vascular endothelial growth factor inhibitor(s)

\section{Declarations}

Availability of data and materials

The datasets used and/or analyzed during the current study are available from the corresponding author on reasonable request.

Ethics approval and consent to participate

This article had Ethics approval and consent to participate.

Consent for publication

We agree that this article will be published in this journal.

Competing interests

There had no competing interests. 
Funding

There had no funding.

Authors' contributions

Bin Zhao, Gong Jian provided the framework and ideas; Wang Shuyue, Chen Mingzhu, Zhang Xinghui, Zhang Lingjian, Jia Min, Shen Zhiwen, Wang Junyan, responsible for data collation and collection; Xu Jing, Zheng Zheng, Wang Mengmeng, Lv Xuanrui, Zong Xiaoyu, Li Hui, responsible for writing articles and collecting documents; Zhou Jin, Meng Tong for review and improvement.

Acknowledgements

Thank all friends help in the process of making and collecting the data, especially for the active cooperation of teachers and students in the process of this survey, and also thank the professor J. Zhao's careful guidance in the process of completing the paper.

\section{References}

1. Ferrara N, Gerber HP, Le Couter J. The biology of VEGF and its receptors. Nat Med. 2003;9(6):669-76.

2. Folkman J. Tumor angiogenesis: therapeutic implications. N Engl J Med. 1971;285(21):1182-6.

3. Eremina V, Jefferson JA, Kowalewska J, Hochster H, Haas M, Weisstuch J, et al. VEGF inhibition and renal thrombotic microangiopathy. N Engl J Med. 2008;358(11):1129-36.

4. Baxi SS, Sherman EJ, Kelly KW, Brown KT, Dematteo RP, Pfister DG. Hemorrhagic pseudoaneurysm in a patient receiving aflibercept for metastatic thyroid cancer. Thyroid: official journal of the American Thyroid Association. 2012;22(5):552-5.

5. Maleux G, Vaninbroukx J, Heye S, van Cutsem E, Oyen R. Aneurysm formation in an angiomyolipoma during bevacizumab combination therapy. Acta oncologica (Stockholm Sweden). 2010;49(6):864-6.

6. Takada M, Yasui T, Oka T, Shioyama W, Kuroda T, Nakai Y,et al. Aortic Dissection and Cardiac Dysfunction Emerged Coincidentally During the Long-Term Treatment with Angiogenesis Inhibitors for Metastatic Renal Cell Carcinoma. Int Heart J. 2018;59(5):1174-9.

7. Oshima Y, Tanimoto T, Yuji K, Tojo A. Association Between Aortic Dissection and Systemic Exposure of Vascular Endothelial Growth Factor Pathway Inhibitors in the Japanese Adverse Drug Event Report Database. Circulation. 2017;135(8):815-7.

8. Aragon-Ching JB, Ning YM, Dahut WL. Acute aortic dissection in a hypertensive patient with prostate cancer undergoing chemotherapy containing bevacizumab. Acta oncologica (Stockholm Sweden). 2008;47(8):1600-1.

9. Funahashi Y, Sassa N, Inada-Inoue M, Ando Y, Matsukawa Y, Gotoh M. Acute aortic dissection in a patient receiving multiple tyrosine kinase inhibitors for 5 years. Aktuelle Urologie. 2014;45(2):132-4. 
10. Yajima K, Koga A, Okumura T, Yamashita K, Isogaki J, Suzuki K, et al. [A Patient with Lung Cancer Experiencing Abdominal Aortic Aneurysm Rupture during Bevacizumab Treatment-Case Report]. Gan to kagaku ryoho Cancer chemotherapy. 2019;46(9):1449-51.

11. Chen G, Ning LJ, Qin Y, Zhao B, Mei D, Li XM. Acute kidney injury following the use of different proton pump inhibitor regimens: A real-world analysis of post-marketing surveillance data. J Gastroenterol Hepatol. 2020. 10.1111/jgh.15151.

12. Hu Y, Gong J, Zhang L, Li X, Li X, Zhao B, et al. Colitis following the use of immune checkpoint inhibitors: A real-world analysis of spontaneous reports submitted to the FDA adverse event reporting system. Int Immunopharmacol. 2020;84:106601.

13. Van Puijenbroek EP, Bate A, Leufkens HG, Lindquist M, Orre R, Egberts AC. A comparison of measures of disproportionality for signal detection in spontaneous reporting systems for adverse drug reactions. Pharmacoepidemiol Drug Saf. 2002;11(1):3-10.

14. Szumilas M. Explaining odds ratios. J Can Acad Child Adolesc Psychiatry. 2010;19(3):227-9.

15. Hauben M, Madigan D, Gerrits CM, Walsh L, Van Puijenbroek EP. The role of data mining in pharmacovigilance. Expert Opin Drug Saf. 2005;4(5):929-48.

16. Edeline J, Laguerre B, Rolland Y, Patard JJ. Aortic dissection in a patient treated by sunitinib for metastatic renal cell carcinoma. Annals of oncology: official journal of the European Society for Medical Oncology. 2010;21(1):186-7.

17. Serrano C, Suárez C, Andreu J, Carles J. Acute aortic dissection during sorafenib-containing therapy. Annals of oncology: official journal of the European Society for Medical Oncology. 2010;21(1):1812.

18. Formiga MN, Fanelli MF. Aortic dissection during antiangiogenic therapy with sunitinib. A case report. Sao Paulo medical journal = Revista paulista de medicina. 2015; 133(3):275-277.

19. Hatem R, Bebawi E, Schampaert E. Potential Sunitinib-Induced Coronary Artery and Aortic Dissections. Can J Cardiol. 2017;33(6):817-30.

20. Xu L, Wang B, Ding W. Abdominal aortic dissection during sorafenib therapy for hepatocellular carcinoma. Clinics research in hepatology gastroenterology. 2017;41(2):24-5.

21. Adachi T, Sato A, Hanaoka D, Aonuma K. Acute aortic dissection with sporadic aortic calcifications during chemotherapy with sunitinib. Journal of vascular surgery cases innovative techniques. 2018;4(2):147.

22. Zenoni D, Beretta FN, Martinelli V, laculli A, Benzoni Fratelli MT, Bonzi D. Aortic dissection after ramucirumab infusion. European journal of hospital pharmacy: science practice. 2020;27(2):117-20.

23. Koda T, Koike J, Masuhara H, Kurihara A, Shiokawa H, Ushigome M, et al. [A Case of Aortoesophageal Fistula Rupture Due to Descending Thoracic Aortic Dissection with Recurrent Colon Cancer during Chemotherapy Containing Bevacizumab]. Gan to kagaku ryoho Cancer chemotherapy. 2016;43(12):1815-7.

\section{Figures}




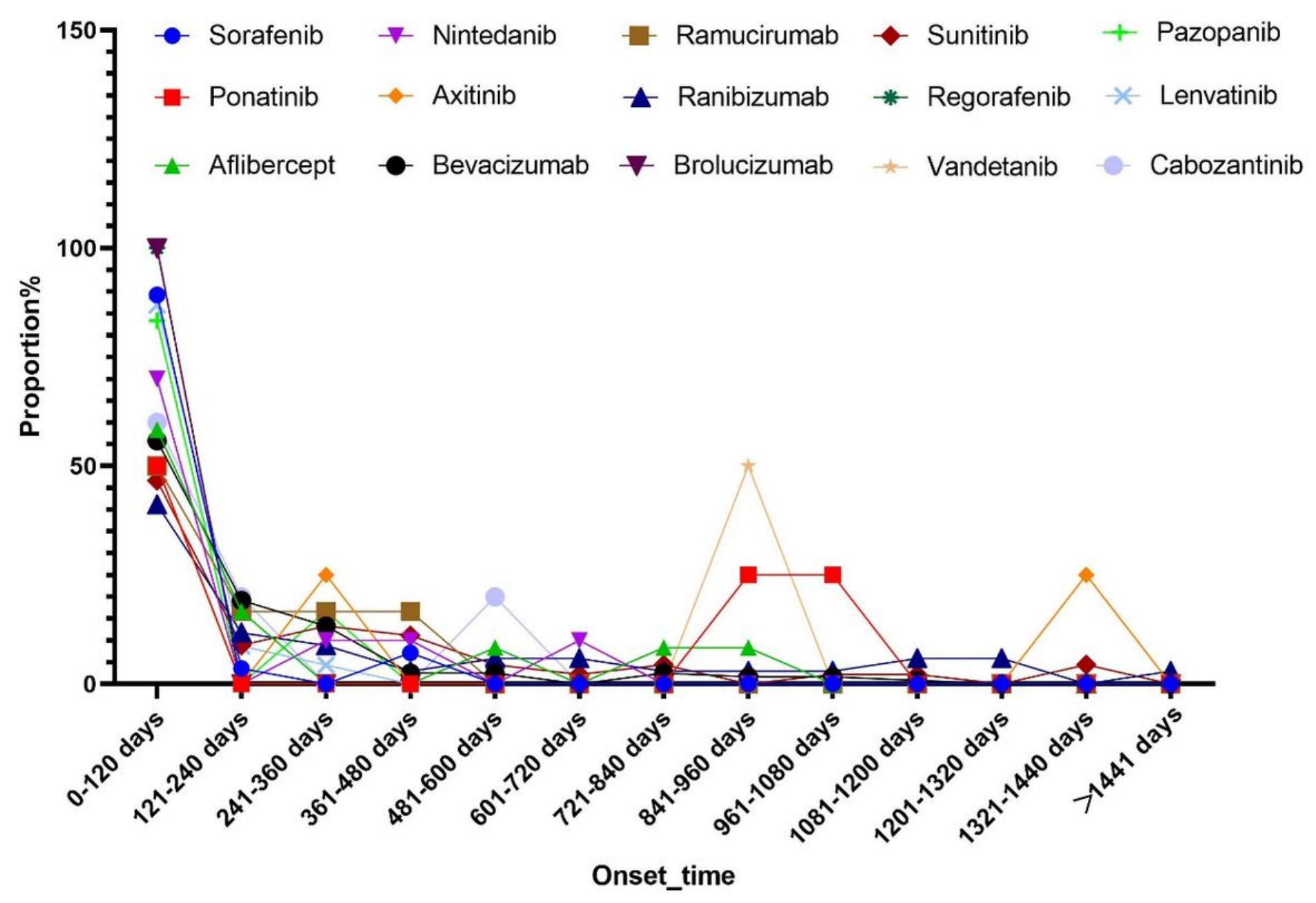

Figure 1

The time to onset of aneurysm and artery dissection for each VPI 


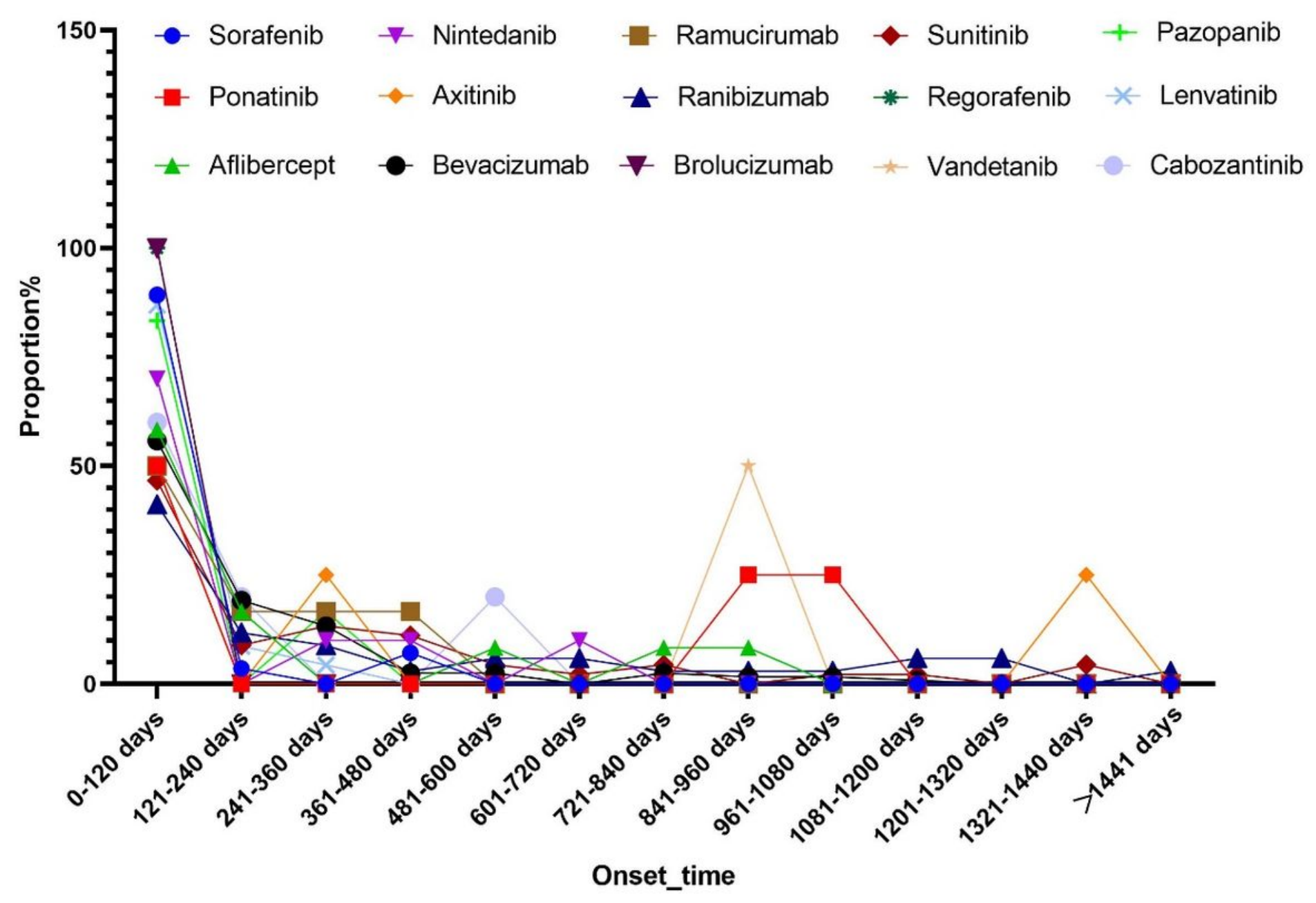

Figure 1

The time to onset of aneurysm and artery dissection for each VPI 


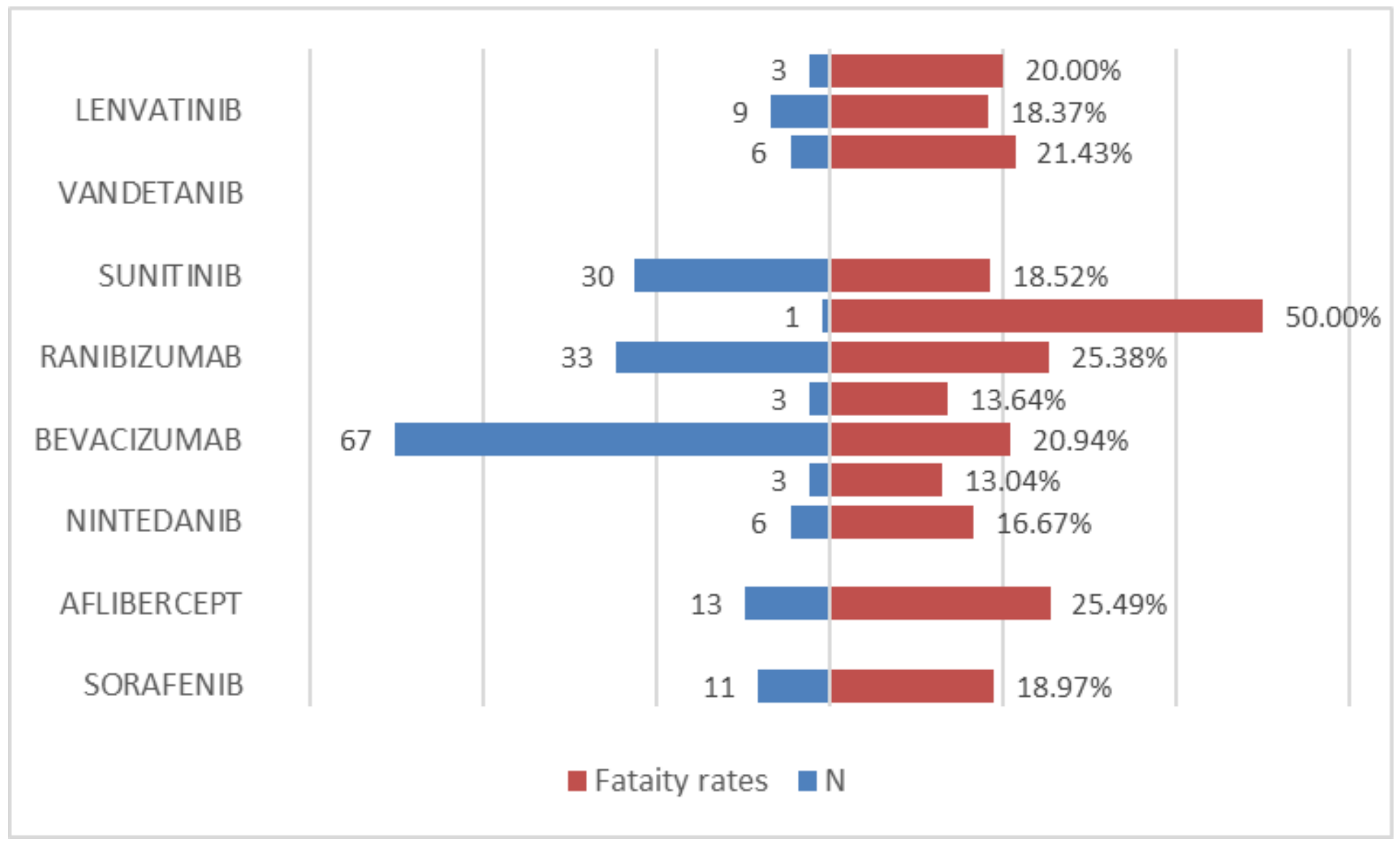

Figure 2

Two-way butterfly diagram of the fatality rates 


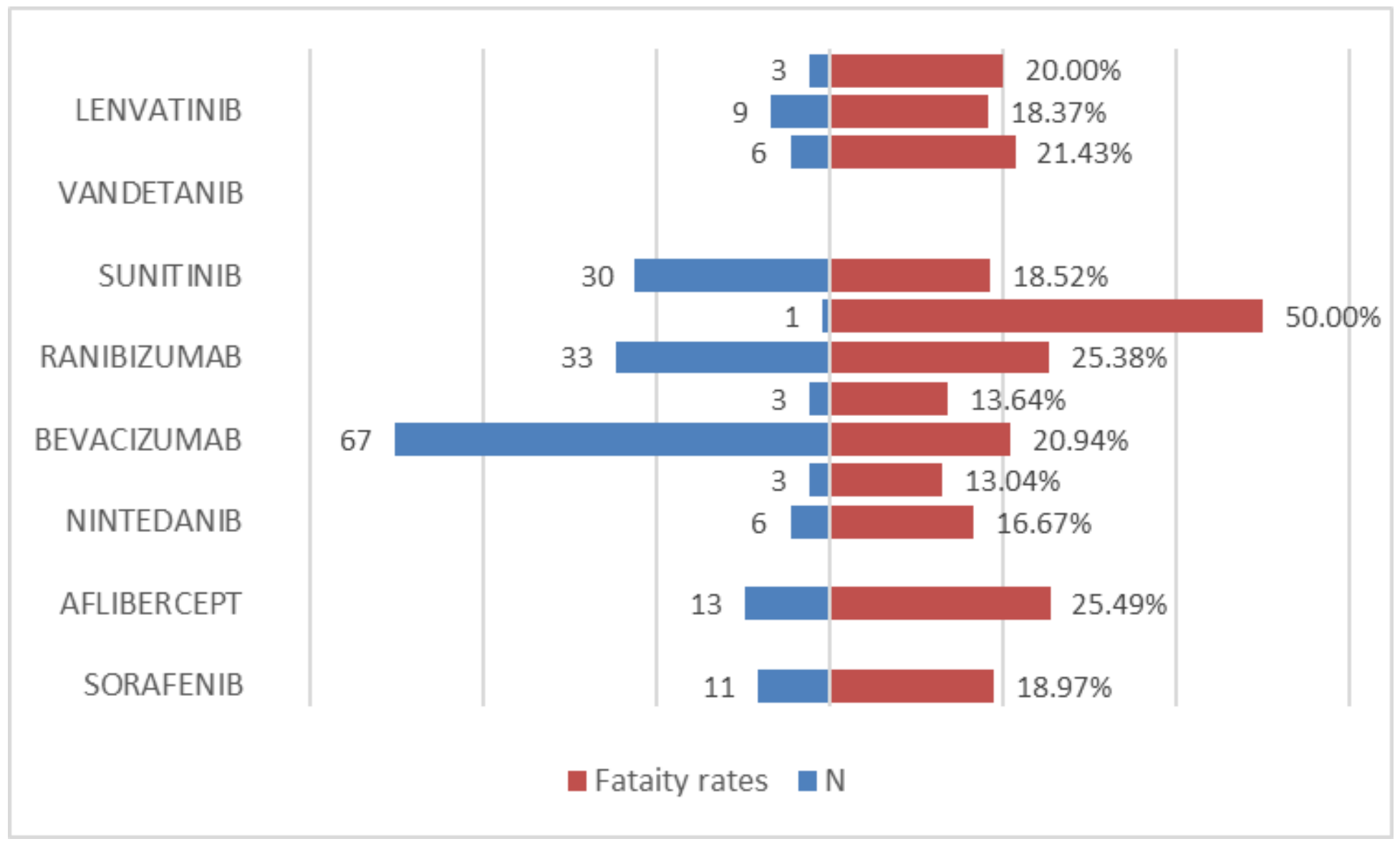

Figure 2

Two-way butterfly diagram of the fatality rates

\section{Supplementary Files}

This is a list of supplementary files associated with this preprint. Click to download.

- supplementarytables.docx

- supplementarytables.docx 